\title{
Modified semi-implicit midpoint rule for nonexpansive mappings
}

\author{
Yonghong Yao ${ }^{1}$, Naseer Shahzad ${ }^{2 *}$ and Yeong-Cheng Liou ${ }^{3,4}$
}

\author{
"Correspondence: \\ nshahzad@kau.edu.sa \\ ${ }^{2}$ Operator Theory and Applications \\ Research Group, Department of \\ Mathematics, King Abdulaziz \\ University, P.O. Box 80203, Jeddah, \\ 21589, Saudi Arabia \\ Full list of author information is \\ available at the end of the article
}

\begin{abstract}
The purpose of the paper is to construct iterative methods for finding the fixed points of nonexpansive mappings. We present a modified semi-implicit midpoint rule with the viscosity technique. We prove that the suggested method converges strongly to a special fixed point of nonexpansive mappings under some different control conditions. Some applications are also included.
\end{abstract}

MSC: $47 \mathrm{~J} 25 ; 47 \mathrm{~N} 20 ; 34 \mathrm{G} 20 ; 65 \mathrm{~J} 15$

Keywords: modified semi-implicit midpoint rule; nonexpansive mapping; strong convergence

\section{Introduction}

The implicit midpoint rule is one of the powerful numerical methods for solving ordinary differential equations and differential algebraic equations. For related works, please refer to [1-9].

For the ordinary differential equation

$$
x^{\prime}=f(t), \quad x(0)=x_{0},
$$

the implicit midpoint rule generates a sequence $\left\{x_{n}\right\}$ by the recursion procedure

$$
x_{n+1}=x_{n}+h f\left(\frac{x_{n}+x_{n+1}}{2}\right), \quad n \geq 0,
$$

where $h>0$ is a stepsize. It is known that if $f: \mathbb{R}^{N} \rightarrow \mathbb{R}^{N}$ is Lipschitz continuous and sufficiently smooth, then the sequence $\left\{x_{n}\right\}$ generated by (1.2) converges to the exact solution of (1.1) as $h \rightarrow 0$ uniformly over $t \in[0, \bar{t}]$ for any fixed $\bar{t}>0$.

If we write the function $f$ in the form $f(t)=g(t)-t$, then differential equation (1.1) becomes $x^{\prime}=g(t)-t$. Then the equilibrium problem associated with the differential equation is the fixed point problem $t=g(t)$.

Based on the above fact, Alghamdi et al. [10] presented the following semi-implicit midpoint rule for nonexpansive mappings:

$$
x_{n+1}=\left(1-\alpha_{n}\right) x_{n}+\alpha_{n} T\left(\frac{x_{n}+x_{n+1}}{2}\right), \quad n \geq 0,
$$

(c) 2015 Yao et al. This article is distributed under the terms of the Creative Commons Attribution 4.0 International License (http://creativecommons.org/licenses/by/4.0/), which permits unrestricted use, distribution, and reproduction in any medium, provided you give appropriate credit to the original author(s) and the source, provide a link to the Creative Commons license, and indicate if changes were made. 
where $\alpha_{n} \in(0,1)$ and $T: H \rightarrow H$ is a nonexpansive mapping. They proved the weak convergence of (1.3) under some additional conditions on $\left\{\alpha_{n}\right\}$.

Furthermore, in [11], Xu et al. used contractions to regularize the semi-implicit midpoint rule (1.3) and presented the following viscosity implicit midpoint rule for nonexpansive mappings:

$$
x_{n+1}=\alpha_{n} Q\left(x_{n}\right)+\left(1-\alpha_{n}\right) T\left(\frac{x_{n}+x_{n+1}}{2}\right), \quad n \geq 0,
$$

where $\alpha_{n} \in(0,1)$ and $Q$ is a contraction.

$\mathrm{Xu}$ et al. [11] showed the following strong convergence theorem.

Theorem 1.1 Let $H$ be a Hilbert space, $C$ be a nonempty, closed, and convex subset of $H$, and $T: C \rightarrow C$ be a nonexpansive mapping such that $\operatorname{Fix}(T) \neq \emptyset$. Let $Q: C \rightarrow C$ be a contraction with coefficient $\alpha \in[0,1)$. Assume that the sequence $\left\{\alpha_{n}\right\}$ satisfies the following three restrictions:

(C1): $\lim _{n \rightarrow \infty} \alpha_{n}=0$;

(C2): $\sum_{n=0}^{\infty} \alpha_{n}=\infty$;

(C3): either $\sum_{n=0}^{\infty}\left|\alpha_{n+1}-\alpha_{n}\right|<\infty$ or $\lim _{n \rightarrow \infty} \frac{\alpha_{n+1}}{\alpha_{n}}=1$.

Then the sequence $\left\{x_{n}\right\}$ generated by (1.4) converges in norm to a fixed point $q$ of $T$, which is also the unique solution of the variational inequality

$$
\langle(I-Q) q, x-q\rangle \geq 0, \quad \forall x \in \operatorname{Fix}(T) .
$$

In other words, $q$ is the unique fixed point of the contraction $P_{\mathrm{Fix}(T)} Q$, that is, $P_{\mathrm{Fix}(T)} Q(q)=q$.

Remark 1.2 The usefulness of (1.4) is that it can be used to find a periodic solution of the time-dependent nonlinear evolution equation (see [11])

$$
\frac{d u}{d t}+A(t) u=g(t, u), \quad t \geq 0
$$

where $A(t)$ is a family of closed linear operators in a Hilbert space $H$ and $g$ maps $\mathbb{R}^{1} \times H$ into $H$.

Remark 1.3 Note that the proof of Theorem 1.1 in [11] is technical. However, Step 6 in the proof of Theorem 1.1 is also complicated.

Fixed point method has attracted so much attention. Now we briefly recall some related historic approaches.

Browder [12] introduced an implicit scheme as follows. Fix $u \in C$ and, for each $t \in(0,1)$, let $x_{t}$ be the unique fixed point in $C$ of the contraction $T_{t}$ which maps $C$ into $C: T_{t} x=$ $t u+(1-t) T x, x \in C$. Browder proved that $s-\lim _{t \downarrow 0} x_{t}=P_{\mathrm{Fix}(T)} u$. That is, the strong limit of $\left\{x_{t}\right\}$ as $t \rightarrow 0^{+}$is the fixed point of $T$ which is nearest from $\operatorname{Fix}(T)$ to $u$.

Halpern [13], on the other hand, introduced an explicit scheme. Again fix a $u \in C$. Then with a sequence $\left\{t_{n}\right\}$ in $(0,1)$ and an arbitrary initial guess $x_{0} \in C$, we can define a sequence $\left\{x_{n}\right\}$ through the recursive formula

$$
x_{n+1}=\alpha_{n} u+\left(1-\alpha_{n}\right) T x_{n}, \quad n \geq 0 .
$$


Lions [14] proved the strong convergence of (1.6) under conditions (C1), (C2) and

$$
\text { (C4): } \lim _{n \rightarrow \infty} \frac{\left|\alpha_{n}-\alpha_{n-1}\right|}{\alpha_{n}^{2}}=0 \text {. }
$$

It is now known that this sequence $\left\{x_{n}\right\}$ converges in norm to the same limit $P_{\mathrm{Fix}(T)} u$ as Browder's implicit scheme if the sequence $\left\{\alpha_{n}\right\}$ satisfies assumptions (C1), (C2), and (C3) above.

Moudafi [15] presented an explicit viscosity method for nonexpansive mappings which generates a sequence $\left\{x_{n}\right\}$ through the iteration process

$$
x_{n+1}=\alpha_{n} Q\left(x_{n}\right)+\left(1-\alpha_{n}\right) T x_{n}, \quad n \geq 0 .
$$

Moudafi proved the strong convergence of (1.7) under conditions (C1), (C2), and

$$
\text { (C5): } \lim _{n \rightarrow \infty} \frac{\left|\alpha_{n}-\alpha_{n-1}\right|}{\alpha_{n} \alpha_{n-1}}=0 \text {. }
$$

Refinements in Hilbert spaces and extensions to Banach spaces were obtained by $\mathrm{Xu}$ [16]. This technique uses (strict) contractions to regularize a nonexpansive mapping for the purpose of selecting a particular fixed point of the nonexpansive mapping, for instance, the fixed point of minimal norm, or of a solution to another variational inequality.

Motivated and inspired by the above work, in this paper we aim to construct a unified iterative algorithm for finding the fixed points of nonexpansive mappings. We present a modified semi-implicit midpoint rule with the viscosity technique for nonexpansive mappings. We prove that the suggested algorithm converges strongly to a special fixed point of nonexpansive mappings under some different conditions. Some applications are also included.

\section{Tools}

\subsection{Some notations}

Let $H$ be a real Hilbert space with inner product $\langle\cdot, \cdot\rangle$ and norm $\|\cdot\|$, respectively. Let $C$ be a nonempty closed convex subset of $H$.

Recall that a mapping $T: C \rightarrow C$ is said to be nonexpansive if

$$
\|T x-T y\| \leq\|x-y\|
$$

for all $x, y \in C$. We use Fix $(T)$ to denote the set of fixed points of $T$.

A mapping $Q: C \rightarrow C$ is said to be contractive if there exists a constant $\alpha \in(0,1)$ such that

$$
\|Q(x)-Q(y)\| \leq \alpha\|x-y\|
$$

for all $x, y \in C$. In this case, $Q$ is called $\alpha$-contraction.

Let $C$ be a nonempty closed convex subset of $H$. For every point $x \in H$, there exists a unique nearest point in $C$ denoted by $P_{C} x$ such that

$$
\left\|x-P_{C} x\right\| \leq\|x-y\|, \quad \forall y \in C .
$$


The mapping $P_{C}$ is called the metric projection of $H$ onto $C$. It is well known that $P_{C}$ is a nonexpansive mapping and is characterized by the following property:

$$
\left\langle x-P_{C} x, y-P_{C} x\right\rangle \leq 0, \quad \forall x \in H, y \in C .
$$

\subsection{Existing algorithm and convergence result}

Let $C$ be a nonempty closed convex subset of a real Hilbert space $H$. Let $Q: C \rightarrow C$ be an $\alpha$-contraction and $T: C \rightarrow C$ be a nonexpansive mapping with $\operatorname{Fix}(T) \neq \emptyset$.

Algorithm 2.1 For given $y_{0} \in C$ arbitrarily, let the sequence $\left\{y_{n}\right\}$ be defined iteratively by the manner

$$
y_{n}=\alpha_{n} Q\left(y_{n}\right)+\left(1-\alpha_{n}\right) T y_{n}, \quad n \geq 0,
$$

where $\left\{\alpha_{n}\right\}$ is a sequence in $(0,1)$.

Theorem 2.2 ([16]) The sequence $\left\{y_{n}\right\}$ generated by (2.2) converges strongly to $q=$ $P_{\mathrm{Fix}(T)} Q(q)$ provided $\lim _{n \rightarrow \infty} \alpha_{n}=0$.

\section{Some lemmas}

The following demiclosedness principles for nonexpansive mappings are well known.

Lemma 3.1 ([17]) Let $C$ be a nonempty closed convex subset of a Hilbert space $H$, and let $T: C \rightarrow C$ be a nonexpansive mapping with $\operatorname{Fix}(T) \neq \emptyset$. Assume that $\left\{y_{n}\right\}$ is a sequence in $C$ such that $y_{n} \rightarrow x^{\dagger}$ and $(I-T) y_{n} \rightarrow 0$. Then $x^{\dagger} \in \operatorname{Fix}(T)$.

Lemma 3.2 ([18]) Let $\left\{x_{n}\right\}$ and $\left\{y_{n}\right\}$ be bounded sequences in a Banach space $E$ and $\left\{\beta_{n}\right\}$ be a sequence in $[0,1]$ with $0<\liminf _{n \rightarrow \infty} \beta_{n} \leq \lim _{\sup _{n \rightarrow \infty}} \beta_{n}<1$. Suppose that $x_{n+1}=(1-$ $\left.\beta_{n}\right) x_{n}+\beta_{n} z_{n}$ for all $n \geq 0$ and $\lim \sup _{n \rightarrow \infty}\left(\left\|z_{n+1}-z_{n}\right\|-\left\|x_{n+1}-x_{n}\right\|\right) \leq 0$. Then $\lim _{n \rightarrow \infty} \| z_{n}-$ $x_{n} \|=0$.

Lemma 3.3 ([19]) Let $\left\{a_{n}\right\}_{n \in \mathbb{N}}$ be a sequence of nonnegative real numbers satisfying the following relation:

$$
a_{n+1} \leq\left(1-\alpha_{n}\right) a_{n}+\alpha_{n} \sigma_{n}+\delta_{n}, \quad n \geq 0,
$$

where

(i) $\left\{\alpha_{n}\right\}_{n \in \mathbb{N}} \subset[0,1]$ and $\sum_{n=1}^{\infty} \alpha_{n}=\infty$;

(ii) $\limsup _{n \rightarrow \infty} \sigma_{n} \leq 0$;

(iii) $\sum_{n=1}^{\infty} \delta_{n}<\infty$.

Then $\lim _{n \rightarrow \infty} a_{n}=0$.

\section{Main results}

In this section, we firstly present the following unified algorithm.

Let $C$ be a nonempty closed convex subset of a real Hilbert space $H$. Let $T: C \rightarrow C$ be a nonexpansive mapping with $\operatorname{Fix}(T) \neq \emptyset$. Let $Q: C \rightarrow C$ be an $\alpha$-contraction. 
Algorithm 4.1 For given $x_{0} \in C$ arbitrarily, let the sequence $\left\{x_{n}\right\}$ be generated by the manner

$$
x_{n+1}=\alpha_{n} Q\left(x_{n}\right)+\beta_{n} x_{n}+\gamma_{n} T\left(\frac{x_{n}+x_{n+1}}{2}\right), \quad n \geq 0
$$

where $\left\{\alpha_{n}\right\} \subset(0,1),\left\{\beta_{n}\right\} \subset[0,1)$, and $\left\{\gamma_{n}\right\} \subset(0,1)$ are three sequences satisfying $\alpha_{n}+\beta_{n}+$ $\gamma_{n}=1$ for all $n \geq 0$.

Remark 4.2 Equation (4.1) is well defined. As a matter of fact, for fixed $u \in C$, we can define a mapping

$$
x \mapsto T_{u} x:=\alpha Q(u)+\beta u+\gamma T\left(\frac{u+x}{2}\right) .
$$

Then we have

$$
\begin{aligned}
\left\|T_{u} x-T_{u} y\right\| & =\gamma\left\|T\left(\frac{u+x}{2}\right)-T\left(\frac{u+y}{2}\right)\right\| \\
& \leq \frac{\gamma}{2}\|x-y\| .
\end{aligned}
$$

This means $T_{u}$ is a contraction with coefficient $\frac{\gamma}{2} \in\left(0, \frac{1}{2}\right)$. Hence, Algorithm 4.1 is well defined.

Now, we show the boundedness of the sequence $\left\{x_{n}\right\}$.

Conclusion 4.3 The sequence $\left\{x_{n}\right\}$ generated by (4.1) is bounded.

Proof Pick any $x^{\dagger} \in \operatorname{Fix}(T)$. From (4.1), we have

$$
\begin{aligned}
\left\|x_{n+1}-x^{\dagger}\right\|= & \| \alpha_{n}\left(Q\left(x_{n}\right)-Q\left(x^{\dagger}\right)\right)+\alpha_{n}\left(Q\left(x^{\dagger}\right)-x^{\dagger}\right)+\beta_{n}\left(x_{n}-x^{\dagger}\right) \\
& +\gamma_{n}\left(T\left(\frac{x_{n}+x_{n+1}}{2}\right)-x^{\dagger}\right) \| \\
\leq & \alpha_{n}\left\|Q\left(x_{n}\right)-Q\left(x^{\dagger}\right)\right\|+\alpha_{n}\left\|Q\left(x^{\dagger}\right)-x^{\dagger}\right\|+\beta_{n}\left\|x_{n}-x^{\dagger}\right\| \\
& +\gamma_{n}\left\|T\left(\frac{x_{n}+x_{n+1}}{2}\right)-x^{\dagger}\right\| \\
\leq & \alpha_{n} \alpha\left\|x_{n}-x^{\dagger}\right\|+\alpha_{n}\left\|Q\left(x^{\dagger}\right)-x^{\dagger}\right\|+\beta_{n}\left\|x_{n}-x^{\dagger}\right\| \\
& +\frac{\gamma_{n}}{2}\left\|x_{n}-x^{\dagger}\right\|+\frac{\gamma_{n}}{2}\left\|x_{n+1}-x^{\dagger}\right\| .
\end{aligned}
$$

It follows that

$$
\begin{aligned}
\left\|x_{n+1}-x^{\dagger}\right\| & \leq \frac{1+\beta_{n}+(2 \alpha-1) \alpha_{n}}{1+\beta_{n}+\alpha_{n}}\left\|x_{n}-x^{\dagger}\right\|+\frac{2 \alpha_{n}}{1+\beta_{n}+\alpha_{n}}\left\|Q\left(x^{\dagger}\right)-x^{\dagger}\right\| \\
& =\left[1-\frac{2(1-\alpha) \alpha_{n}}{1+\beta_{n}+\alpha_{n}}\right]\left\|x_{n}-x^{\dagger}\right\|+\frac{2(1-\alpha) \alpha_{n}}{1+\beta_{n}+\alpha_{n}} \frac{1}{1-\alpha}\left\|Q\left(x^{\dagger}\right)-x^{\dagger}\right\| \\
& \leq \max \left\{\left\|x_{n}-x^{\dagger}\right\|, \frac{1}{1-\alpha}\left\|Q\left(x^{\dagger}\right)-x^{\dagger}\right\|\right\} .
\end{aligned}
$$


By induction, we can deduce

$$
\left\|x_{n}-x^{\dagger}\right\| \leq \max \left\{\left\|x_{0}-x^{\dagger}\right\|, \frac{1}{1-\alpha}\left\|Q\left(x^{\dagger}\right)-x^{\dagger}\right\|\right\} .
$$

Hence, $\left\{x_{n}\right\}$ is bounded. This completes the proof.

Now we give the following result.

Theorem 4.4 The sequence $\left\{x_{n}\right\}$ generated by (4.1) converges strongly to $q=P_{\mathrm{Fix}(T)} Q(q)$ provided $\left\{\alpha_{n}\right\}$ satisfies (C1)-(C3) (as stated in Theorem 1.1) and $\left\{\beta_{n}\right\}$ satisfies

$$
\text { (C6): } \lim _{n \rightarrow \infty} \frac{\beta_{n}}{\alpha_{n}}=0 \text {. }
$$

Proof Set $y_{n+1}=\alpha_{n} Q\left(y_{n}\right)+\left(1-\alpha_{n}\right) T\left(\frac{y_{n}+y_{n+1}}{2}\right)$ for all $n$. Then we have

$$
\begin{aligned}
\left\|x_{n+1}-y_{n+1}\right\|= & \| \alpha_{n}\left(Q\left(x_{n}\right)-Q\left(y_{n}\right)\right)+\beta_{n}\left(x_{n}-T\left(\frac{y_{n}+y_{n+1}}{2}\right)\right) \\
& +\gamma_{n}\left(T\left(\frac{x_{n}+x_{n+1}}{2}\right)-T\left(\frac{y_{n}+y_{n+1}}{2}\right)\right) \| \\
\leq & \alpha \alpha_{n}\left\|x_{n}-y_{n}\right\|+\beta_{n}\left\|x_{n}-T\left(\frac{y_{n}+y_{n+1}}{2}\right)\right\|+\frac{\gamma_{n}}{2}\left\|x_{n}-y_{n}\right\| \\
& +\frac{\gamma_{n}}{2}\left\|x_{n+1}-y_{n+1}\right\| .
\end{aligned}
$$

It is obvious that $\left\{x_{n}\right\}$ and $\left\{y_{n}\right\}$ are bounded by Conclusion 4.3. Hence, we deduce from (4.2) that

$$
\begin{aligned}
\left\|x_{n+1}-y_{n+1}\right\| & \leq\left[1-\frac{2(1-\alpha) \alpha_{n}}{2-\gamma_{n}}\right]\left\|x_{n}-y_{n}\right\|+\beta_{n} M_{1} \\
& =\left(1-\sigma_{n}\right)\left\|x_{n}-y_{n}\right\|+\sigma_{n} \frac{\beta_{n}}{\sigma_{n}} M_{1},
\end{aligned}
$$

where $\sigma_{n}=\frac{2(1-\alpha) \alpha_{n}}{2-\gamma_{n}}$ and $M_{1}$ is a constant such that $\sup _{n}\left\{2\left\|x_{n}-T\left(\frac{y_{n}+y_{n+1}}{2}\right)\right\|\right\} \leq M_{1}$. Note that $\limsup _{n \rightarrow \infty} \frac{\beta_{n}}{\sigma_{n}} \leq 0$. Apply Lemma 3.3 to (4.3) to conclude that $\left\|x_{n+1}-y_{n+1}\right\| \rightarrow 0$. Consequently, $x_{n} \rightarrow q=P_{\mathrm{Fix}(T)} Q(q)$ according to Theorem 1.1. This completes the proof.

Remark 4.5 The proof of Theorem 4.4 is very simple.

Remark 4.6 In (4.1), if we choose $\beta_{n} \equiv 0$ for all $n$, then (4.1) is reduced to (1.4). Thus, our Algorithm 4.1 includes Algorithm 1.4 as a special case, and Theorem 1.1 is also a special case of our Theorem 4.4.

Next, we can define the following algorithm.

Algorithm 4.7 For given $y_{0} \in C$ arbitrarily, let the sequence $\left\{y_{n}\right\}$ be defined iteratively by the manner

$$
y_{n}=\alpha_{n} Q\left(y_{n}\right)+\beta_{n} y_{n}+\gamma_{n} T y_{n}, \quad n \geq 0,
$$

where $\left\{\alpha_{n}\right\},\left\{\beta_{n}\right\}$, and $\left\{\gamma_{n}\right\}$ are the same sequences as stated in Algorithm 4.1. 
Proposition 4.8 The sequence $\left\{y_{n}\right\}$ generated by (4.4) converges strongly to $q=P_{\operatorname{Fix}(T)} Q(q)$ provided $\lim _{n \rightarrow \infty} \alpha_{n}=0$.

In fact, we can rewrite (4.4) as $y_{n}=\frac{\alpha_{n}}{1-\beta_{n}} Q\left(y_{n}\right)+\left(1-\frac{\alpha_{n}}{1-\beta_{n}}\right) T y_{n}$ for all $n$. Thus, Proposition 4.8 can be deduced from Theorem 2.2.

Next we use Proposition 4.8 to show the convergence analysis of Algorithm 4.1 under other control conditions.

Let the sequences $\left\{x_{n}\right\}$ and $\left\{y_{n}\right\}$ be generated by (4.1) and (4.4), respectively. Note that the sequences $\left\{x_{n}\right\}$ and $\left\{y_{n}\right\}$ are all bounded. First, we have the following estimation:

$$
\begin{aligned}
\left\|x_{n+1}-y_{n}\right\| & =\left\|\alpha_{n}\left(Q\left(x_{n}\right)-Q\left(y_{n}\right)\right)+\beta_{n}\left(x_{n}-y_{n}\right)+\gamma_{n}\left(T\left(\frac{x_{n}+x_{n+1}}{2}\right)-T y_{n}\right)\right\| \\
& \leq \alpha_{n} \alpha\left\|x_{n}-y_{n}\right\|+\beta_{n}\left\|x_{n}-y_{n}\right\|+\gamma_{n} \frac{\left\|x_{n}-y_{n}\right\|}{2}+\gamma_{n} \frac{\left\|x_{n+1}-y_{n}\right\|}{2} .
\end{aligned}
$$

It follows that

$$
\begin{aligned}
\left\|x_{n+1}-y_{n}\right\| & \leq\left[1-\frac{2(1-\alpha) \alpha_{n}}{1+\alpha_{n}+\beta_{n}}\right]\left\|x_{n}-y_{n}\right\| \\
& \leq\left[1-\frac{2(1-\alpha) \alpha_{n}}{1+\alpha_{n}+\beta_{n}}\right]\left\|x_{n}-y_{n-1}\right\|+\left\|y_{n}-y_{n-1}\right\| .
\end{aligned}
$$

It is easily seen that if $\sum_{n=0}^{\infty} \alpha_{n}=\infty$ and $\lim _{n \rightarrow \infty} \frac{\left\|y_{n}-y_{n-1}\right\|}{\alpha_{n}}=0$, then we get $\lim _{n \rightarrow \infty} \| x_{n+1}-$ $y_{n} \|=0$ by Lemma 3.3. Consequently, $x_{n} \rightarrow q=P_{\mathrm{Fix}(T)} Q(q)$ provided $\lim _{n \rightarrow \infty} \alpha_{n}=0$.

Next, we estimate $\left\|y_{n}-y_{n-1}\right\|$. From (4.4), we have

$$
\begin{aligned}
\left\|y_{n}-y_{n-1}\right\|= & \| \alpha_{n}\left(Q\left(y_{n}\right)-Q\left(y_{n-1}\right)\right)+\left(\alpha_{n}-\alpha_{n-1}\right) Q\left(y_{n-1}\right)+\beta_{n}\left(y_{n}-y_{n-1}\right) \\
& +\left(\beta_{n}-\beta_{n-1}\right) y_{n-1}+\gamma_{n}\left(T y_{n}-T y_{n-1}\right)+\left(\gamma_{n}-\gamma_{n-1}\right) T y_{n-1} \| \\
\leq & \left(\alpha \alpha_{n}+\beta_{n}+\gamma_{n}\right)\left\|y_{n}-y_{n-1}\right\|+\left|\alpha_{n}-\alpha_{n-1}\right|\left\|Q\left(y_{n-1}\right)\right\| \\
& +\left|\beta_{n}-\beta_{n-1}\right|\left\|y_{n-1}\right\|+\left|\gamma_{n}-\gamma_{n-1}\right|\left\|T y_{n-1}\right\| .
\end{aligned}
$$

Hence,

$$
\begin{aligned}
\frac{\left\|y_{n}-y_{n-1}\right\|}{\alpha_{n}} \leq & \frac{\left|\alpha_{n}-\alpha_{n-1}\right|}{(1-\alpha) \alpha_{n}^{2}}\left(\left\|Q\left(y_{n-1}\right)\right\|+\left\|T y_{n-1}\right\|\right) \\
& +\frac{\left|\beta_{n}-\beta_{n-1}\right|}{(1-\alpha) \alpha_{n}^{2}}\left(\left\|y_{n-1}\right\|+\left\|T y_{n-1}\right\|\right) .
\end{aligned}
$$

If $\lim _{n \rightarrow \infty} \frac{\left|\alpha_{n}-\alpha_{n-1}\right|}{\alpha_{n}^{2}}=\lim _{n \rightarrow \infty} \frac{\left|\beta_{n}-\beta_{n-1}\right|}{\alpha_{n}^{2}}=0$, we derive that $\lim _{n \rightarrow \infty} \frac{\left\|y_{n}-y_{n-1}\right\|}{\alpha_{n}}=0$. So, we obtain immediately the following theorem.

Theorem 4.9 Assume that $\left\{\alpha_{n}\right\}$ satisfies $(\mathrm{C} 1),(\mathrm{C} 2)$, and (C4) and $\left\{\beta_{n}\right\}$ satisfies

$$
\text { (C7): } \lim _{n \rightarrow \infty} \frac{\left|\beta_{n}-\beta_{n-1}\right|}{\alpha_{n}^{2}}=0 \text {. }
$$

Then the sequence $\left\{x_{n}\right\}$ generated by (4.1) converges strongly to $q=P_{\mathrm{Fix}(T)} Q(q)$. 
Remark 4.10 Note that conditions (C1), (C2), and (C4) were presented by Lions in [14]. At the same time, (C7) is different from (C6). In fact, we can choose $\beta_{n}=\beta \in(0,1)$ in (C7).

Next, we will give another control condition instead of (C4) and (C7).

Theorem 4.11 Assume that $\left\{\alpha_{n}\right\}$ satisfies $(\mathrm{C} 1)$ and $(\mathrm{C} 2)$ and $\left\{\beta_{n}\right\}$ satisfies

$$
\text { (C8): } 0<\liminf _{n \rightarrow \infty} \beta_{n} \leq \limsup _{n \rightarrow \infty} \beta_{n}<1
$$

and

$$
\text { (C9): } \lim _{n \rightarrow \infty}\left(\beta_{n+1}-\beta_{n}\right)=0 \text {. }
$$

Then the sequence $\left\{x_{n}\right\}$ generated by (4.1) converges strongly to $q=P_{\operatorname{Fix}(T)} Q(q)$.

Proof From Conclusion 4.3, we can choose a constant $M$ such that

$$
\sup _{n}\left\{\frac{3}{1-\beta_{n}}\left(\left\|Q\left(x_{n}\right)\right\|+\left\|T\left(\frac{x_{n}+x_{n+1}}{2}\right)\right\|+\left\|x_{n}-T\left(\frac{x_{n}+x_{n+1}}{2}\right)\right\|\right)\right\} \leq M .
$$

Set $y_{n}=\frac{x_{n+1}-\beta_{n} x_{n}}{1-\beta_{n}}$ for all $n \geq 0$. Thus, we have

$$
\begin{aligned}
y_{n+1}-y_{n}= & \frac{x_{n+2}-\beta_{n+1} x_{n+1}}{1-\beta_{n+1}}-\frac{x_{n+1}-\beta_{n} x_{n}}{1-\beta_{n}} \\
= & \frac{\alpha_{n+1} Q\left(x_{n+1}\right)+\left(1-\alpha_{n+1}-\beta_{n+1}\right) T\left(\frac{x_{n+1}+x_{n+2}}{2}\right)}{1-\beta_{n+1}} \\
& -\frac{\alpha_{n} Q\left(x_{n}\right)+\left(1-\alpha_{n}-\beta_{n}\right) T\left(\frac{x_{n}+x_{n+1}}{2}\right)}{1-\beta_{n}} \\
= & \frac{\alpha_{n+1}}{1-\beta_{n+1}}\left(Q\left(x_{n+1}\right)-Q\left(x_{n}\right)\right) \\
& +\frac{1-\alpha_{n+1}-\beta_{n+1}}{1-\beta_{n+1}}\left[T\left(\frac{x_{n+1}+x_{n+2}}{2}\right)-T\left(\frac{x_{n}+x_{n+1}}{2}\right)\right] \\
& +\left(\frac{\alpha_{n+1}}{1-\beta_{n+1}}-\frac{\alpha_{n}}{1-\beta_{n}}\right)\left(Q\left(x_{n}\right)-T\left(\frac{x_{n}+x_{n+1}}{2}\right)\right) .
\end{aligned}
$$

It follows that

$$
\begin{aligned}
\left\|y_{n+1}-y_{n}\right\| \leq & \left(\frac{\alpha_{n+1}}{1-\beta_{n+1}}+\frac{\alpha_{n}}{1-\beta_{n}}\right)\left\|Q\left(x_{n}\right)-T\left(\frac{x_{n}+x_{n+1}}{2}\right)\right\| \\
& +\frac{1-\alpha_{n+1}-\beta_{n+1}}{1-\beta_{n+1}}\left[\frac{\left\|x_{n+1}-x_{n}\right\|}{2}+\frac{\left\|x_{n+2}-x_{n+1}\right\|}{2}\right] \\
& +\frac{\alpha \alpha_{n+1}}{1-\beta_{n+1}}\left\|x_{n+1}-x_{n}\right\| .
\end{aligned}
$$

From (4.1), we have

$$
\begin{aligned}
\left\|x_{n+2}-x_{n+1}\right\|= & \| \alpha_{n+1}\left(Q\left(x_{n+1}\right)-Q\left(x_{n}\right)\right)+\left(\alpha_{n+1}-\alpha_{n}\right) Q\left(x_{n}\right) \\
& +\beta_{n+1}\left(x_{n+1}-x_{n}\right)+\left(\beta_{n+1}-\beta_{n}\right)\left(x_{n}-T\left(\frac{x_{n}+x_{n+1}}{2}\right)\right)
\end{aligned}
$$




$$
\begin{aligned}
& \quad+\left(1-\alpha_{n+1}-\beta_{n+1}\right)\left(T\left(\frac{x_{n+1}+x_{n+2}}{2}\right)-T\left(\frac{x_{n}+x_{n+1}}{2}\right)\right) \\
& +\left(\alpha_{n}-\alpha_{n+1}\right) T\left(\frac{x_{n}+x_{n+1}}{2}\right) \| \\
& \leq \alpha \alpha_{n+1}\left\|x_{n+1}-x_{n}\right\|+\left(\alpha_{n+1}+\alpha_{n}\right)\left\|Q\left(x_{n}\right)\right\|+\beta_{n+1}\left\|x_{n+1}-x_{n}\right\| \\
& \quad+\left(1-\alpha_{n+1}-\beta_{n+1}\right)\left(\frac{\left\|x_{n+1}-x_{n}\right\|}{2}+\frac{\left\|x_{n+2}-x_{n+1}\right\|}{2}\right) \\
& \quad+\left(\alpha_{n}+\alpha_{n+1}\right)\left\|T\left(\frac{x_{n}+x_{n+1}}{2}\right)\right\|+\left|\beta_{n+1}-\beta_{n}\right|\left\|x_{n}-T\left(\frac{x_{n}+x_{n+1}}{2}\right)\right\| .
\end{aligned}
$$

It follows that

$$
\begin{aligned}
\left\|x_{n+2}-x_{n+1}\right\| \leq & {\left[1-\frac{2(1-\alpha) \alpha_{n+1}}{1+\alpha_{n+1}+\beta_{n+1}}\right]\left\|x_{n+1}-x_{n}\right\| } \\
& +\frac{2\left(\alpha_{n+1}+\alpha_{n}\right)}{1+\alpha_{n+1}+\beta_{n+1}}\left(\left\|Q\left(x_{n}\right)\right\|+\left\|T\left(\frac{x_{n}+x_{n+1}}{2}\right)\right\|\right) \\
& +\frac{2\left|\beta_{n+1}-\beta_{n}\right|}{1+\alpha_{n+1}+\beta_{n+1}}\left\|x_{n}-T\left(\frac{x_{n}+x_{n+1}}{2}\right)\right\| \\
\leq & \left\|x_{n+1}-x_{n}\right\|+M\left(\alpha_{n}+\alpha_{n+1}+\left|\beta_{n+1}-\beta_{n}\right|\right) .
\end{aligned}
$$

Substitute (4.6) into (4.5) to get

$$
\left\|y_{n+1}-y_{n}\right\| \leq\left[1-\frac{(1-\alpha) \alpha_{n+1}}{1-\beta_{n+1}}\right]\left\|x_{n+1}-x_{n}\right\|+2 M\left(\alpha_{n+1}+\alpha_{n}+\left|\beta_{n+1}-\beta_{n}\right|\right) .
$$

Hence,

$$
\limsup _{n \rightarrow \infty}\left(\left\|y_{n+1}-y_{n}\right\|-\left\|x_{n+1}-x_{n}\right\|\right) \leq 0 .
$$

This together with Lemma 3.2 implies that

$$
\lim _{n \rightarrow \infty}\left\|y_{n}-x_{n}\right\|=0
$$

Note that

$$
y_{n}-x_{n}=\frac{x_{n+1}-x_{n}}{1-\beta_{n}}
$$

So,

$$
\lim _{n \rightarrow \infty}\left\|x_{n+1}-x_{n}\right\|=0 .
$$

Again, from (4.1), we have

$$
\begin{aligned}
\left\|x_{n}-T x_{n}\right\| \leq & \left\|x_{n}-x_{n+1}\right\|+\left\|x_{n+1}-T x_{n}\right\| \\
\leq & \left\|x_{n}-x_{n+1}\right\|+\alpha_{n}\left\|Q\left(x_{n}\right)-T x_{n}\right\|+\beta_{n}\left\|x_{n}-T x_{n}\right\| \\
& +\left(1-\alpha_{n}-\beta_{n}\right) \frac{1}{2}\left\|x_{n}-x_{n+1}\right\| .
\end{aligned}
$$


It follows that

$$
\left\|x_{n}-T x_{n}\right\| \leq \frac{\alpha_{n}}{1-\beta_{n}}\left\|Q\left(x_{n}\right)-T x_{n}\right\|+\frac{3-\alpha_{n}-\beta_{n}}{2\left(1-\beta_{n}\right)}\left\|x_{n+1}-x_{n}\right\| .
$$

This together with (C1) and (4.7) implies that

$$
\lim _{n \rightarrow \infty}\left\|x_{n}-T x_{n}\right\|=0
$$

Next, we prove that

$$
\limsup _{n \rightarrow \infty}\left\langle q-Q(q), q-x_{n}\right\rangle \leq 0
$$

where $q \in \operatorname{Fix}(T)$ is the unique fixed point of the contraction $P_{\mathrm{Fix}(T)} Q$, that is, $q=$ $P_{\mathrm{Fix}(T)} Q(q)$.

Since $\left\{x_{n}\right\}$ is bounded, there exists a subsequence $\left\{x_{n_{i}}\right\}$ of $\left\{x_{n}\right\}$ such that $\left\{x_{n_{i}}\right\}$ converges weakly to a point $\breve{x}$ and

$$
\begin{aligned}
& \limsup _{n \rightarrow \infty}\left\langle P_{\operatorname{Fix}(T)} Q(q)-Q(q), P_{\operatorname{Fix}(T)} Q(q)-x_{n}\right\rangle \\
& \quad=\lim _{i \rightarrow \infty}\left\langle P_{\operatorname{Fix}(T)} Q(q)-Q(q), P_{\operatorname{Fix}(T)} Q(q)-x_{n_{i}}\right\rangle .
\end{aligned}
$$

By Lemma 3.1 and (4.8), we deduce $\breve{x} \in \operatorname{Fix}(T)$. This together with (2.1) implies that

$$
\begin{aligned}
& \limsup _{n \rightarrow \infty}\left\langle P_{\mathrm{Fix}(T)} Q(q)-Q(q), P_{\mathrm{Fix}(T)} Q(q)-x_{n}\right\rangle \\
& \quad=\lim _{i \rightarrow \infty}\left\langle P_{\mathrm{Fix}(T)} Q(q)-Q(q), P_{\mathrm{Fix}(T)} Q(q)-x_{n_{i}}\right\rangle \\
& \quad=\left\langle P_{\operatorname{Fix}(T)} Q(q)-Q(q), P_{\mathrm{Fix}(T)} Q(q)-\breve{x}\right\rangle \\
& \quad \leq 0 .
\end{aligned}
$$

Finally, we prove that $x_{n} \rightarrow q$. From (4.1), we have

$$
\begin{aligned}
\left\|x_{n+1}-q\right\|^{2}= & \alpha_{n}\left\langle Q\left(x_{n}\right)-Q(q), x_{n+1}-q\right\rangle+\alpha_{n}\left\langle Q(q)-q, x_{n+1}-q\right\rangle \\
& +\left(1-\alpha_{n}-\beta_{n}\right)\left\langle T\left(\frac{x_{n}+x_{n+1}}{2}\right)-q, x_{n+1}-q\right\rangle \\
& +\beta_{n}\left\langle x_{n}-q, x_{n+1}-q\right\rangle \\
\leq & \alpha_{n} \alpha\left\|x_{n}-q\right\|\left\|x_{n+1}-q\right\|+\alpha_{n}\left\langle Q(q)-q, x_{n+1}-q\right\rangle \\
& +\left(1-\alpha_{n}-\beta_{n}\right) \frac{1}{2}\left(\left\|x_{n}-q\right\|+\left\|x_{n+1}-q\right\|\right)\left\|x_{n+1}-q\right\| \\
& +\beta_{n}\left\|x_{n}-q\right\|\left\|x_{n+1}-q\right\| \\
\leq & \frac{1+\beta_{n}+(2 \alpha-1) \alpha_{n}}{4}\left\|x_{n}-q\right\|^{2}+\frac{3-\beta_{n}-(3-2 \alpha) \alpha_{n}}{4}\left\|x_{n+1}-q\right\|^{2} \\
& +\alpha_{n}\left|Q(q)-q, x_{n+1}-q\right\rangle .
\end{aligned}
$$


It follows that

$$
\begin{aligned}
\left\|x_{n+1}-q\right\|^{2} \leq & {\left[1-\frac{4(1-\alpha) \alpha_{n}}{1+\beta_{n}+(3-2 \alpha) \alpha_{n}}\right]\left\|x_{n}-q\right\|^{2} } \\
& +\frac{4 \alpha_{n}}{1+\beta_{n}+(3-2 \alpha) \alpha_{n}}\left\langle Q(q)-q, x_{n+1}-q\right\rangle .
\end{aligned}
$$

Apply Lemma 3.3 and (4.9) to (4.11) to deduce that $x_{n} \rightarrow q$. This completes the proof.

Remark 4.12 Note that condition (C8) has been used in a large number of references. Theorems 4.4, 4.9, and 4.11 demonstrate the strong convergence of Algorithm 4.1 under different control conditions on parameters $\left\{\alpha_{n}\right\}$ and $\left\{\beta_{n}\right\}$. Our algorithm and results provide a unified framework for the class problem of algorithmic approach to the fixed point of nonlinear operators.

\section{Applications}

\subsection{Application to variational inequalities}

Let $C$ be a nonempty closed convex subset of a real Hilbert space $H$. Let $A: H \rightarrow H$ be a single-valued monotone operator such that $C \subset \operatorname{dom}(A)$. Now we consider the following variational inequality:

$$
\left\langle A x^{*}, x-x^{*}\right\rangle \geq 0, \quad x \in C
$$

It is known that (5.1) is equivalent to the fixed point problem, for any $\lambda>0$,

$$
P_{C}(I-\lambda A) x^{*}=x^{*}
$$

If $A$ is Lipschitzian and $\alpha$-inverse-strongly monotone, then $P_{C}(I-\lambda A)$ is nonexpansive provided $0<\lambda<2 \alpha$. Thus, we can get the following theorem.

Theorem 5.1 Let $C$ be a nonempty closed convex subset of a real Hilbert space H. Let $A: H \rightarrow H$ be a Lipschitzian and $\alpha$-inverse-strongly monotone operator. Let $Q: C \rightarrow C$ be a contraction. Assume (5.1) is solvable. Let $\left\{x_{n}\right\}$ be a sequence generated by the manner

$$
x_{n+1}=\alpha_{n} Q\left(x_{n}\right)+\beta_{n} x_{n}+\gamma_{n} P_{C}(I-\lambda A)\left(\frac{x_{n}+x_{n+1}}{2}\right), \quad n \geq 0,
$$

where $\lambda \in(0,2 \alpha)$ and $\left\{\alpha_{n}\right\}$ and $\left\{\beta_{n}\right\}$ satisfy one of the following conditions: (C1), (C2), (C4), and $(\mathrm{C} 7)$ or $(\mathrm{C} 1),(\mathrm{C} 2),(\mathrm{C} 3)$, and $(\mathrm{C} 6)$ or $(\mathrm{C} 1),(\mathrm{C} 2),(\mathrm{C} 8)$, and $(\mathrm{C} 9)$. Then the sequence $\left\{x_{n}\right\}$ converges strongly to a solution $x^{*}$ of $(5.1)$ which is also a solution to the variational inequality

$$
\left\langle(I-Q) x^{*}, x-x^{*}\right\rangle \geq 0, \quad x \in A^{-1}(0) .
$$

\subsection{Application to hierarchical minimization}

Consider the following hierarchical minimization problem:

$$
\min _{x \in S_{0}} \psi_{1}(x)
$$


where $S_{0}:=\arg \min _{x \in H} \psi_{0}(x)$ and $\psi_{0}, \psi_{1}$ are two lower semi-continuous convex functions from $H$ to $\mathbb{R}$. Assume that $S_{0} \neq \emptyset$. Set $S=\arg \min _{x \in S_{0}} \psi_{1}(x)$ and assume $S \neq \emptyset$.

Assume that $\psi_{0}$ and $\psi_{1}$ are differentiable and their gradients satisfy the Lipschitz continuity conditions

$$
\left\|\nabla \psi_{0}(x)-\nabla \psi_{0}(y)\right\| \leq L_{0}\|x-y\|, \quad\left\|\nabla \psi_{1}(x)-\nabla \psi_{1}(y)\right\| \leq L_{1}\|x-y\|
$$

for all $x, y \in H$. Note that the Lipschitz continuity (5.5) implies that $\nabla \psi_{i}$ is $\frac{1}{L_{i}}$-inversestrongly monotone. Consequently, $\left(I-\gamma_{i} \nabla \psi_{i}\right)$ is nonexpansive provided $0<\gamma_{i}<2 / L_{i}$ and $S_{0}=\operatorname{Fix}\left(\left(I-\gamma_{1} \nabla \psi_{0}\right)\right)$.

The optimality condition for $x^{*} \in S_{0}$ to be a solution of the hierarchical minimization (5.4) is the variational inequality

$$
x^{*} \in S_{0}, \quad\left\langle\nabla \psi_{1}\left(x^{*}\right), x-x^{*}\right| \geq 0, \quad x \in S_{0} .
$$

Hence, we have the following theorem.

Theorem 5.2 Assume that the hierarchical minimization problem (5.4) is solvable. Assume (5.5) and $0<\gamma_{i}<2 / L_{i}$. Let $Q: C \rightarrow C$ be a contraction. Define a sequence $\left\{x_{n}\right\}$ by the manner

$$
x_{n+1}=\alpha_{n} Q\left(x_{n}\right)+\beta_{n} x_{n}+\gamma_{n} P_{S_{0}}\left(I-\lambda \nabla \psi_{1}\right)\left(\frac{x_{n}+x_{n+1}}{2}\right), \quad n \geq 0,
$$

where $\left\{\alpha_{n}\right\}$ and $\left\{\beta_{n}\right\}$ satisfy one of the following conditions: (C1), (C2), (C4), and (C7) or $(\mathrm{C} 1),(\mathrm{C} 2),(\mathrm{C} 3)$, and $(\mathrm{C} 6)$ or $(\mathrm{C} 1),(\mathrm{C} 2),(\mathrm{C} 8)$, and (C9). Then the sequence $\left\{x_{n}\right\}$ converges strongly to a solution $x^{*}$ of (5.6) which is also a solution to the variational inequality

$$
\left\langle(I-Q) x^{*}, x-x^{*}\right\rangle \geq 0, \quad x \in S
$$

\subsection{Periodic solution of a nonlinear evolution equation}

Consider the time-dependent nonlinear equation of evolution in $H$ given by

$$
\frac{d u}{d t}+A(t) u=f(t, u), \quad t \geq 0
$$

where $A(t)$ is a family of closed linear operators in a Hilbert space $H$ and $f$ maps $\mathbb{R}^{1} \times H$ into $H$.

We assume that $A(t)$ and $f(t, u)$ are periodic in $t$ with a common period $\xi>0$.

An interesting result on the existence of periodic solutions of equation (5.8) is due to Browder [20].

Theorem 5.3 Suppose that $A(t)$ and $f(t, u)$ are periodic in $T$ of period $\xi>0$ and satisfy the following assumptions:

(i) For each $t$ and each pair $u, v \in H$,

$$
\operatorname{Re}\langle f(t, u)-f(t, v), u-v\rangle \leq 0 .
$$


(ii) For each $t$ and each $u \in D(A(t)), \operatorname{Re}\langle A(t) u, u\rangle \geq 0$.

(iii) There exists a mild solution $u$ of equation (5.1) on $\mathbb{R}^{+}$for each initial value $v \in H$.

(iv) There exists some $R>0$ such that

$$
\begin{gathered}
\operatorname{Re}\langle f(t, u), u\rangle<0 \\
\text { for }\|u\|=R \text { and all } t \in[0, \xi] .
\end{gathered}
$$

Then there exists an element $v$ of $H$ with $\|v\|<R$ such that the mild solution of equation (5.8) with the initial condition $u(0)=v$ is of period $\xi$.

Define a mapping $T: H \rightarrow H$ by

$$
T v=u(\xi), \quad v \in H
$$

where $u$ solves (5.8) with $u(0)=v$.

Then each fixed point of $T$ corresponds to a periodic solution of equation (5.8) with period $\xi$. Since

$$
\begin{aligned}
\frac{1}{2} \frac{d}{d t}\left\{\|u(t)\|^{2}\right\} & =-\operatorname{Re}\langle A(t) u(t), u(t)\rangle+\operatorname{Re}\langle f(t, u(t)), u(t)\rangle \\
& \leq \operatorname{Re}\langle f(t, u(t)), u(t)\rangle,
\end{aligned}
$$

we see that for any value of $t$ in $[0, \xi]$ for which $\|u(t)\|=R$, we have $\frac{d}{d t}\left\{\|u(t)\|^{2}\right\}<0$. Hence, $\|u(\xi)\| \leq R$, and $T$ maps the closed ball $B:=\{v \in H:\|v\| \leq R\}$ into itself.

At the same time, we note that $T$ is nonexpansive. As a matter of fact, if $v$ and $v_{1}$ are two elements of $B, u(t)$ and $u_{1}(t)$ are the corresponding mild solutions, we have

$$
\begin{aligned}
\frac{1}{2} \frac{d}{d t}\left\{\left\|u(t)-u_{1}(t)\right\|^{2}\right\}= & -\operatorname{Re}\left\langle A(t)\left(u(t)-u_{1}(t)\right), u(t)-u_{1}(t)\right\rangle \\
& +\operatorname{Re}\left\langle f(t, u(t))-f\left(t, u_{1}(t)\right), u(t)-u_{1}(t)\right\rangle
\end{aligned}
$$$$
\leq 0 \text {. }
$$

Hence, $\left\|u(\xi)-u_{1}(\xi)\right\| \leq\left\|u(0)-u_{1}(0)\right\|$, i.e., $\left\|T v-T v_{1}\right\| \leq\left\|v-v_{1}\right\|$.

Consequently, $T$ has a fixed point which we denote by $v$, and the corresponding solution $u$ of (5.8) with the initial condition $u(0)=v$ is a desired periodic solution of (5.8) with period $\xi$. In other words, to find a periodic solution $u$ of (5.8) is equivalent to finding a fixed point of $T$.

Thus, our method is applicable to (5.8). It turns out that under one of the following conditions: (C1), (C2), (C4), and (C7) or (C1), (C2), (C3), and (C6) or (C1), (C2), (C8), and (C9), the sequence $\left\{v_{n}\right\}$ generated by the manner

$$
v_{n+1}=\alpha_{n} Q\left(v_{n}\right)+\beta_{n} v_{n}+\gamma_{n} T\left(\frac{v_{n}+v_{n+1}}{2}\right), \quad n \geq 0
$$

converges strongly to a fixed point $v$ of $T$, and the mild solution of (5.8) with the initial value $u(0)=\xi$ is a periodic solution of (5.8). 


\subsection{Fredholm integral equation}

Consider a Fredholm integral equation of the form

$$
x(t)=g(t)+\int_{0}^{t} F(t, s, x(s)) d s, \quad t \in[0,1]
$$

where $g$ is a continuous function on $[0,1]$ and $F:[0,1] \times[0,1] \times \mathbb{R} \rightarrow \mathbb{R}$ is continuous. Note that if $F$ satisfies the Lipschitz continuity condition

$$
|F(t, s, x)-F(t, s, y)| \leq|x-y|, \quad t, s \in[0,1], x, y \in \mathbb{R},
$$

then equation (5.10) has at least one solution in $L^{2}[0,1]$ (see [14]).

Define a mapping $T: L^{2}[0,1] \rightarrow L^{2}[0,1]$ by

$$
(T x)(t)=g(t)+\int_{0}^{t} F(t, s, x(s)) d s, \quad t \in[0,1]
$$

It is easily seen that $T$ is nonexpansive. In fact, we have, for $x, y \in L^{2}[0,1]$,

$$
\begin{aligned}
\|T x-T y\|^{2} & =\int_{0}^{1}|T x(t)-T y(t)|^{2} d t \\
& =\int_{0}^{1}\left|\int_{0}^{1}(F(t, s, x(s))-F(t, s, y(s))) d s\right|^{2} d t \\
& \leq \int_{0}^{1}\left|\int_{0}^{2}\right| x(s)-y(s)|d s|^{2} d t \\
& \leq \int_{0}^{1}|x(s)-y(s)|^{2} d s \\
& =\|x-y\|^{2} .
\end{aligned}
$$

This means that to find the solution of integral equation (5.10) is reduced to finding a fixed point of the nonexpansive mapping $T$ in the Hilbert space $L^{2}[0,1]$.

Initiating with any function $y_{0} \in L^{2}[0,1]$, define a sequence of functions $\left\{y_{n}\right\}$ in $L^{2}[0,1]$ by

$$
y_{n+1}=\alpha_{n} Q\left(y_{n}\right)+\beta_{n} y_{n}+\gamma_{n} T\left(\frac{y_{n}+y_{n+1}}{2}\right), \quad n \geq 0 .
$$

Then the sequence $\left\{y_{n}\right\}$ converges strongly in $L^{2}[0,1]$ to the solution of integral equation (5.10) under one of the following conditions: (C1), (C2), (C4), and (C7) or (C1), (C2), (C3), and (C6) or (C1), (C2), (C8), and (C9).

\section{Competing interests}

The authors declare that they have no competing interests. 


\section{Author details}

'Department of Mathematics, Tianjin Polytechnic University, Tianjin, 300387, China. ${ }^{2}$ Operator Theory and Applications Research Group, Department of Mathematics, King Abdulaziz University, P.O. Box 80203, Jeddah, 21589, Saudi Arabia. ${ }^{3}$ Department of Information Management, Cheng Shiu University, Kaohsiung, 833, Taiwan. ${ }^{4}$ Center for General Education, Kaohsiung Medical University, Kaohsiung, 807, Taiwan.

\section{Acknowledgements}

This article was funded by the Deanship of Scientific Research (DSR), King Abdulaziz University, Jeddah. The authors, therefore, acknowledge with thanks DSR for technical and financial support.

\section{Received: 22 May 2015 Accepted: 28 August 2015 Published online: 17 September 2015}

\section{References}

1. Hofer, E: A partially implicit method for large stiff systems of ODEs with only few equations introducing small time-constants. SIAM J. Numer. Anal. 13, 645-663 (1976)

2. Bader, G, Deuflhard, P: A semi-implicit mid-point rule for stiff systems of ordinary differential equations. Numer. Math. 41, 373-398 (1983)

3. Deuflhard, P: Recent progress in extrapolation methods for ordinary differential equations. SIAM Rev. 27(4), 505-535 (1985)

4. Auzinger, W, Frank, R: Asymptotic error expansions for stiff equations: an analysis for the implicit midpoint and trapezoidal rules in the strongly stiff case. Numer. Math. 56, 469-499 (1989)

5. Bayreuth, A: The implicit midpoint rule applied to discontinuous differential equations. Computing 49, 45-62 (1992)

6. Schneider, C: Analysis of the linearly implicit mid-point rule for differential-algebra equations. Electron. Trans. Numer. Anal. 1, 1-10 (1993)

7. Somalia, S, Davulcua, S: Implicit midpoint rule and extrapolation to singularly perturbed boundary value problems. Int. J. Comput. Math. 75(1), 117-127 (2000)

8. Somalia, S: Implicit midpoint rule to the nonlinear degenerate boundary value problems. Int. J. Comput. Math. 79(3), 327-332 (2002)

9. Hrabalova, J, Tomasek, P: On stability regions of the modified midpoint method for a linear delay differential equation. Adv. Differ. Equ. 2013, 177 (2013)

10. Alghamdi, MA, Alghamdi, MA, Shahzad, N, Xu, HK: The implicit midpoint rule for nonexpansive mappings. Fixed Point Theory Appl. 2014, 96 (2014)

11. Xu, HK, Alghamdi, MA, Shahzad, N: The viscosity technique for the implicit midpoint rule of nonexpansive mappings in Hilbert spaces. Fixed Point Theory Appl. 2015, 41 (2015)

12. Browder, FE: Convergence of approximation to fixed points of nonexpansive nonlinear mappings in Hilbert spaces. Arch. Ration. Mech. Anal. 24, 82-90 (1967)

13. Halpern, B: Fixed points of nonexpanding maps. Bull. Am. Math. Soc. 73, 591-597 (1967)

14. Lions, PL: Approximation de points fixes de contractions. C. R. Acad. Sci., Ser. A-B Paris 284, 1357-1359 (1977)

15. Moudafi, A: Viscosity approximation methods for fixed-points problems. J. Math. Anal. Appl. 241, 46-55 (2000)

16. Xu, HK: Viscosity approximation methods for nonexpansive mappings. J. Math. Anal. Appl. 298, $279-291$ (2004)

17. Goebel, K, Kirk, WA: Topics in Metric Fixed Point Theory. Cambridge Studies in Advanced Mathematics, vol. 28. Cambridge University Press, Cambridge (1990)

18. Suzuki, T: Strong convergence theorems for infinite families of nonexpansive mappings in general Banach spaces. Fixed Point Theory Appl. 2005, 103-123 (2005)

19. Xu, HK: Iterative algorithms for nonlinear operators. J. Lond. Math. Soc. 66, 240-256 (2002)

20. Browder, FE: Existence of periodic solutions for nonlinear equations of evolution. Proc. Natl. Acad. Sci. USA 53 1100-1103 (1965)

\section{Submit your manuscript to a SpringerOpen ${ }^{\ominus}$ journal and benefit from:}

- Convenient online submission

- Rigorous peer review

- Immediate publication on acceptance

Open access: articles freely available online

- High visibility within the field

- Retaining the copyright to your article 